\title{
Sufficient Conditions and Sensitivity Analysis for Optimal Bang-Bang Control Problems with State Constraints
}

\author{
Helmut Maurer ${ }^{1}$ and Georg Vossen ${ }^{2}$ \\ 1 Institut für Numerische und Angewandte Mathematik, Westfälische Wilhelms-Universität \\ Münster, Einsteinstrasse 62, D-48149 Münster, Germany \\ maurer@math.uni-muenster.de \\ 2 Fraunhofer-Institut für Lasertechnik, Steinbachstr. 15, 52074 Aachen, Germany \\ georg.vosseneilt.fraunhofer. de
}

\begin{abstract}
Bang-bang control problems subject to a state inequality constraint are considered. It is shown that the control problem induces an optimization problem, where the optimization vector assembles the switching and junction times for bang-bang and boundary arcs. Second order sufficient conditions (SSC) for the state-constrained control problem are given which require that SSC for the induced optimization problem are satisfied and a generalized strict bang-bang property holds at switching and junction times. This type of SSC ensures solution differentiability of optimal solutions under parameter perturbations and allows to compute parametric sensitivity derivatives. A numerical algorithm is presented that simultaneously determines a solution candidate, performs the second-order test and computes parametric sensitivity derivatives. We illustrate the algorithm with two state-constrained optimal control problems in biomedicine.
\end{abstract}

\section{Introduction}

Second-order sufficient optimality conditions (SSC) for bang-bang controls without state constraints have been derived in Agrachev, Stefani and Zezza [1] on the basis of an induced optimization problem where the control process is optimized with respect to the unknown switching times of the bang-bang control. The equivalence of this type of SSC with a different form of SSC obtained earlier in the literature has recently been shown in [20[2123]. Numerical methods for the verification of optimization based SSC have been developed in Maurer, Büskens, Kim and Kaya [18] using the so-called arc-parameterization method where the arc-lengths of the bang-bang arcs are optimized. Basic ideas for a sensitivity analysis of bang-bang controls may be found in Kim, Maurer [11].

The purpose of this paper is to extend the results and techniques in [18 11] to bangbang control problems with a state constraint. In Sect.2] we review the necessary conditions for an optimal control problem with a state constraint of order one. The regularity conditions in assumptions (A1), (A2) allow to determine the multiplier associated with the state constraint. In Sect. 3 we formulate an induced optimization problem where the 
optimization vector assembles the switching times of bang-bang arcs, resp., the entryand exit-times (junction times) of boundary arcs, and the free final time. The crucial point in this optimization approach is the fact that the optimal control is given in feedback form along boundary arcs.

Based on second-order sufficient conditions (SSC) for the induced optimization problem, we give SSC for the control problem which require the strict bang-bang property in assumption (A4) and the strict complementarity in (A5). In Sect. 4 the arc-parameterization method from [18] is extended to incorporate boundary arcs. The main result on sensitivity analysis is given in Sect.5. We include a formula for the sensitivity derivatives of the switching and junction times which can be implemented into the routine NUDOCCCS developed in [4]; cf. also [5]6]. In Sect. 6] we discuss the drug displacement problem in [22] in the light of the SSC presented in Theorem 2 In Sect.7] we determine the optimal control in a two-compartment model for cancer therapy [12], when a state constraint on the number of tumor cells is imposed.

\section{Optimal Bang-Bang Control Problems with a State Constraint}

Let $x(t) \in \mathbb{R}^{n}$ denote the state variable and $u(t) \in \mathbb{R}$ the control variable at time $t \in\left[0, t_{f}\right]$, where the final time $t_{f}>0$ is either fixed or free. For simplicity, the control is assumed to be scalar. The following autonomous optimal control problem with control variable appearing linearly will be denoted by $(O C)$ : determine a measurable control function $u:\left[0, t_{f}\right] \rightarrow \mathbb{R}$ and a terminal time $t_{f}>0$ such that the pair of functions $(x(\cdot), u(\cdot))$ minimizes the cost functional of Mayer type

$$
J\left(x, u, t_{f}\right):=g\left(x\left(t_{f}\right), t_{f}\right)
$$

subject to the constraints in the interval $\left[0, t_{f}\right]$,

$$
\begin{gathered}
\dot{x}(t)=f(x(t), u(t))=f_{0}(x(t))+f_{1}(x(t)) u(t), \\
x(0)=x_{0}, \varphi\left(x\left(t_{f}\right), t_{f}\right)=0, \\
u_{\min } \leq u(t) \leq u_{\max },
\end{gathered}
$$

and the scalar state inequality constraint

$$
S(x(t)) \leq 0 \text { for } 0 \leq t \leq t_{f} .
$$

The functions $g: \mathbb{R}^{n} \times \mathbb{R} \rightarrow \mathbb{R}, f_{0}, f_{1}: \mathbb{R}^{n} \rightarrow \mathbb{R}^{n}, \varphi: \mathbb{R}^{n} \times \mathbb{R} \rightarrow \mathbb{R}^{r}, 0 \leq r \leq n$, and $S: \mathbb{R}^{n} \rightarrow \mathbb{R}$ are assumed to be twice continuously differentiable. The state constraint is assumed to be of order one [9]16], i.e., the total time derivative of the function $S(x(t))$ contains the control explicitly,

$$
S^{1}(x, u):=S_{x}(x)\left(f_{0}(x)+f_{1}(x) u\right)=a(x)+b(x) u,
$$

where $b(x)=S_{x}(x) f_{1}(x) \not \equiv 0$. Here and in the sequel, partial derivatives are denoted by subscripts. A subinterval $\left[\tau_{1}, \tau_{2}\right] \subset\left[0, t_{f}\right]$ is called an interior arc if $S(x(t))<0$ holds on $\left(\tau_{1}, \tau_{2}\right)$. The interval $\left[\tau_{1}, \tau_{2}\right]$ is called a boundary arc if $S(x(t)) \equiv 0$ holds for all 
$t \in\left[\tau_{1}, \tau_{2}\right]$. If $\left[\tau_{1}, \tau_{2}\right]$ is maximal with this property, then $\tau_{1}$ is called entry-time and $\tau_{2}$ is called exit-time of the boundary arc; $\tau_{1}, \tau_{2}$ are also called junction times. The following assumption is a standard regularity condition for a boundary arc [9]15]17].

$$
b(x(t)) \neq 0 \forall t \in\left[\tau_{1}, \tau_{2}\right] .
$$

Under this assumption, the boundary control on a boundary arc is determined by the equation $S^{1}(x, u)=a(x)+b(x) u=0$ as the feedback expression

$$
u_{b}(x)=-a(x) / b(x), u(t)=u_{b}(x(t)) .
$$

The following assumption will be needed to determine the multiplier associated with the state constraint explicitly.

(A2) The boundary control lies in the interior of the control region:

$$
u_{\min }<u(t)=u_{b}(x(t))<u_{\max } \forall t \in\left[\tau_{1}, \tau_{2}\right] .
$$

Assumptions (A1) and (A2) allow us to formulate first order necessary conditions of Pontryagin's minimum principle in a computationally convenient form. We recall from [917] that the Lagrange multiplier associated with the state constraint (5) is a measure that is represented by a function $\mu$ of bounded variation. Using (A1) and (A2), it has been shown in [16]17]15]14] that the measure has a Radon-Nikodym derivative $\eta$ which allows to write the following adjoint equation (11) in differential form. In the direct adjoining approach [9]17], the augmented Pontryagin or Hamiltonian function is defined by

$$
H(x, u, \lambda, \mu)=\lambda f(x, u)+\eta S(x)=\lambda f_{0}(x)+\lambda f_{1}(x) u+\eta S(x),
$$

where the adjoint variable $\lambda \in \mathbb{R}^{n}$ is a row vector and $\eta$ is the multiplier associated with the state constraint.

Suppose now that $\bar{u}:\left[0, \bar{t}_{f}\right] \rightarrow\left[u_{\min }, u_{\max }\right]$ is an optimal control with optimal final time $\bar{t}_{f}$ and corresponding trajectory $\bar{x}:\left[0, \bar{t}_{f}\right] \rightarrow \mathbb{R}^{n}$. Assume that (A1), (A2) hold and the state constraint (5) is not active at $t=0$ and $t=\bar{t}_{f}$,

$$
S(\bar{x}(0))<0 \text { and } S\left(\bar{x}\left(\bar{t}_{f}\right)\right)<0 .
$$

In the sequel, we will use the junction theorem in [16], Corollary 5.2 (ii), where it was shown that the adjoint variables are continuous at junction times provided that the state constraint is of first order and the control is discontinuous at junctions. Note that the latter property follows from (A2). Then there exist an absolutely continuous (a.c.) adjoint function $\lambda:\left[0, \bar{t}_{f}\right] \rightarrow \mathbb{R}^{n}$, a piecewise a.c. multiplier function $\eta:\left[0, \bar{t}_{f}\right] \rightarrow \mathbb{R}$ and a multiplier $\rho \in \mathbb{R}^{r}$ (row vector) such that the following conditions hold a.e. on $\left[0, \bar{t}_{f}\right]$ :

$$
\begin{aligned}
& \dot{\lambda}(t)=-H_{x}(\bar{x}(t), \bar{u}(t), \lambda(t), \eta(t)), \\
& \lambda\left(\bar{t}_{f}\right)=l_{x}\left(\bar{x}\left(\bar{t}_{f}\right), \bar{t}_{f}, \rho\right), \\
& \left.H(\bar{x}(t), \bar{u}(t), \lambda(t), \eta(t))\right|_{t=\bar{t}_{f}}+l_{t_{f}}\left(\bar{x}\left(\bar{t}_{f}\right), \bar{t}_{f}, \rho\right)=0, \text { if } t_{f} \text { is free, } \\
& H(\bar{x}(t), \bar{u}(t), \lambda(t), \eta(t))=\min \left\{H(\bar{x}(t), u, \lambda(t), \eta(t)) \mid u \in\left[u_{\min }, u_{\max }\right]\right\}, \\
& \eta(t) \geq 0, \eta(t)=0, \text { if } S(x(t))<0,
\end{aligned}
$$


where $l\left(x, t_{f}, \rho\right):=(g+\rho \varphi)\left(x, t_{f}\right)$ is the endpoint Lagrangian function. The switching function is defined by

$$
\sigma(x, \lambda):=H_{u}=\lambda f_{1}(x), \sigma(t)=\sigma(x(t), \lambda(t)) .
$$

On interior arcs with $S(x(t))<0$ the minimum condition 14 yields the control law

$$
u(t)=\left\{\begin{array}{l}
u_{\min }, \text { if } \sigma(t)>0, \\
u_{\max }, \text { if } \sigma(t)<0 .
\end{array}\right.
$$

The switching times of the control are zeroes of the switching function. A singular arc occurs if the switching function $\sigma(t)$ vanishes identically on an interval $I_{\text {sing }} \subset\left[0, \bar{t}_{f}\right]$. In this paper, we assume that the optimal control does not contain singular arcs.

Along a boundary arc $\left[\tau_{1}, \tau_{2}\right]$, assumption (A2) requires that the control takes values in the interior of the control set. Hence, the minimum condition (14) implies

$$
\sigma(t)=\lambda(t) f_{1}(x(t))=0 \forall t \in\left[\tau_{1}, \tau_{2}\right] .
$$

This relation can be interpreted as the property that a boundary control behaves formally like a singular control, a fact that was exploited in [16] to obtain junction theorems. By differentiating (18) and using the adjoint equation (11) we find the following explicit representation of the multiplier $\eta=\eta(x, \lambda)$ in (11) (cf. [17|14]),

$$
\eta(x, \lambda)=\lambda\left[\left(f_{1}\right)_{x}(x) f\left(x, u_{b}(x)\right)-f_{x}\left(x, u_{b}(x)\right) f_{1}(x)\right] / b(x),
$$

where $u_{b}(x(t))$ is the boundary control (7). In short, the multiplier is given by the Lie bracket $\eta(x, \lambda)=\lambda\left[f_{1}, f\right]$; we then set $\eta(t)=\eta(x(t), \lambda(t))$.

\section{Induced Optimization Problem and Second Order Sufficient Conditions}

In order to transcribe the control problem into a finite-dimensional optimization problem, we make the following assumption:

(A3) The optimal control has finitely many bang-bang and boundary arcs.

Under assumptions (A1)-(A3), the optimal control problem can be transcribed into an optimization problem in the following way. We assume that the structure of the optimal control, i.e., the sequence of finitely many bang-bang and boundary arcs, is known. Let $t_{j}, j=1, \ldots, s$, be the switching and junction times which are ordered as

$$
0=: t_{0}<t_{1}<\ldots<t_{j}<\ldots<t_{s}<t_{s+1}:=t_{f} .
$$

For simplicity, assume that there exists only a single boundary arc $\left[\tau_{1}, \tau_{2}\right]=\left[t_{k}, t_{k+1}\right]$ with an index $1 \leq k \leq s$. Then $\left[0, t_{k}\right]$ and $\left[t_{k+1}, t_{f}\right]$ are the interior arcs. By assumption, in every interval $I_{j}:=\left[t_{j-1}, t_{j}\right]$ there exists a function $u^{j}(x)$ with the property that the optimal control is given by

$$
u(t)=u^{j}(x(t)), t_{j-1} \leq t \leq t_{j}, \quad(j=1, \ldots, s, s+1) .
$$


The interval $I_{k+1}$ then represents the boundary arc. The function $u^{j}(x)$ is either the constant value of the bang-bang control on interior arcs or the boundary control $u^{k+1}(x)=$ $u_{b}(x)=-a(x) / b(x)$.

Consider now the optimization variable $z:=\left(t_{1}, \ldots, t_{s+1}\right)^{*} \in \mathbb{R}^{s+1}$ with $t_{s+1}:=t_{f}$ in case of a free final time, resp., $z:=\left(t_{1}, \ldots, t_{s}\right)^{*} \in \mathbb{R}^{s}$ for fixed final time $t_{f}$, where the asterisk denotes the transpose. Denote by $x(t ; z)$ the absolutely continuous solution of the ODE system

$$
\dot{x}(t)=f\left(x(t), u^{j}(x(t))\right), t_{j-1} \leq t \leq t_{j},
$$

with initial condition $x(0)=x_{0}$. Then the control problem $(O C)$ can be reformulated as the following finite-dimensional optimization problem $(O P)$ with equality constraints:

$$
\begin{array}{ll}
\operatorname{Min} & G(z):=g\left(x\left(t_{s+1} ; z\right), t_{s+1}\right) \\
\text { s.t. } & \Phi(z):=\varphi\left(x\left(t_{s+1} ; z\right), t_{s+1}\right)=0, \\
& \mathscr{S}(z):=S\left(x\left(t_{k} ; z\right)\right)=0 .
\end{array}
$$

The last equation arises from the entry-condition for the boundary arc. We consider the Lagrangian for the induced optimization problem $(O P)$ in normal form,

$$
\mathscr{L}(z, \rho, \beta)=G(z)+\rho \Phi(z)+\beta \mathscr{S}(z),
$$

with multipliers $\rho \in \mathbb{R}^{r}$ (row vector) and $\beta \in \mathbb{R}$. First order necessary and second order sufficient conditions (SSC) for (23) are well known in the literature. In the following theorem, we consider control problems with free final time which involve the optimization vector $z \in \mathbb{R}^{s+1}$.

Theorem 1. SSC FOR THE INDUCED OPTIMIZATION PROBLEM.

Let $\bar{z}$ be feasible for the optimization problem (23). Suppose there exist multipliers $\rho \in$ $\mathbb{R}^{r}$ and $\beta \in \mathbb{R}$ such that the following three conditions hold:

(a) $\operatorname{rank}\left[\Phi_{z}(\bar{z}) \mid \mathscr{S}_{z}(\bar{z})\right]=r+1$,

(b) $L_{z}(\bar{z}, \rho, \beta)=0$,

(c) $v^{*} L_{z z}(\bar{z}, \rho, \beta) v>0$ for all $v \in \mathbb{R}^{s+1} \backslash\{0\}$ with $\Phi_{z}(\bar{z}) v=0, \mathscr{S}_{z}(\bar{z}) v=0$.

Then $\bar{z}$ is a strict local minimizer of the optimization problem $(O P)$.

Arguments similar to those in [18,23] reveal that the first order conditions in part (a) and (b) of Theorem 1 are closely related to those in 11-13) involving the adjoint function $\lambda(t)$. Namely, using the multiplier $\rho$ in the Lagrangian (24), we define the adjoint function $\lambda(t)$ through the transversality condition $\lambda\left(\bar{t}_{f}\right)=(g+\rho \varphi)_{x}\left(\bar{x}\left(\bar{t}_{f}\right), \bar{t}_{f}\right)$ in (12) and the adjoint equation (11) where the multiplier $\eta$ is given in (19). However, on the boundary arc $\left[t_{k}, t_{k+1}\right]$ there exists another multiplier $\eta^{1}$ and an adjoint function $\lambda^{1}(t)$ which correspond to the so-called indirect adjoining approach [9]17]. Here, the Hamiltonian is defined by $H^{1}=\lambda^{1} f(x, u)+\eta^{1} S^{1}(x, u)$ with the function $S^{1}(x, u)$ given in (6). Then the adjoint equation is $\dot{\lambda}^{1}=-H_{x}^{1}$ and the multiplier $\eta^{1}$ is determined via the equation $H_{u}^{1}=0$ as $\eta^{1}\left(x, \lambda^{1}\right)=-\lambda^{1} f_{u}\left(x, u_{b}(x)\right) / b(x)$. The multiplier $\beta$ in the Lagrangian (24) yields the jump condition $\lambda^{1}\left(t_{k}+\right)=\lambda^{1}\left(t_{k}-\right)-\beta S_{x}\left(x\left(t_{k}\right)\right)$ at the entrytime $t_{k}$. Moreover, one can show the relation $\beta=\int_{t_{k}}^{t_{k+1}} \eta(t) d t \geq 0$. 
For bang-bang control problems without state constraints, it was shown in [1 120 21 23] that one further needs the so-called strict bang-bang property to obtain SSC for the bangbang control problem. The following assumption gives an extension of the strict bangbang property to control problems with state constraints.

(A4) (a) on interior arcs for $j=1, \ldots, k-1, k+2, \ldots, s$ :

$$
\sigma\left(t_{j}\right)=0, \dot{\sigma}\left(t_{j}\right)\left(u\left(t_{j}^{-}\right)-u\left(t_{j}^{+}\right)\right)>0, \sigma(t) \neq 0 \text { for } t \neq t_{j} .
$$

(b) at the entry-time $t_{k}$ and exit-time $t_{k+1}$ of the boundary arc:

$$
\dot{\sigma}\left(t_{k}-\right)\left(u\left(t_{k}^{-}\right)-u\left(t_{k}^{+}\right)\right)>0, \dot{\sigma}\left(t_{k+1}^{+}\right)\left(u\left(t_{k+1}^{-}\right)-u\left(t_{k+1}^{+}\right)\right)>0 .
$$

Finally, we need the following strict complementarity condition:

(A5) The multiplier $\eta(t)$ satisfies $\eta(t)>0 \forall t \in\left[t_{k}, t_{k+1}\right]$.

Note that assumptions (A4) and (A5) have also been used in [13 14] to construct a local field of extremals near boundary arcs. This has enabled the authors to prove sufficient conditions (SSC) which correspond to the following form of SSC. Detailed proofs will be given elsewhere.

Theorem 2. SSC FOR THE STATE-CONSTRAINED CONTROL PROBLEM.

Let $\bar{u}$ be a feasible control for the control problem (1)-(5) which has finitely many switching and junction times $\bar{t}_{j}, j=1, \ldots, s$, and let $\bar{x}$ be the corresponding trajectory. Suppose there exists an adjoint function $\lambda:\left[0, t_{f}\right] \rightarrow \mathbb{R}^{n}$ and a multiplier $\rho \in \mathbb{R}^{r}$ such that assumptions (A1)-(A5) hold with multiplier function $\eta:\left[0, t_{f}\right] \rightarrow \mathbb{R}$ defined by (19). Suppose further that the vector $\bar{z}=\left(\bar{t}_{1}, \ldots, \bar{t}_{s}, \bar{t}_{s+1}\right)^{*} \in \mathbb{R}^{s+1}, \bar{t}_{s+1}=\bar{t}_{f}$, satisfies the SSC in Theorem 1 Then the control $\bar{u}$ provides a strict strong minimum for the control problem $(O C)$.

\section{Numerical Methods for Solving the Induced Optimization Problem}

In this section, we shall extend the arc-parameterization method in [10 18] to solve state-constrained control problems. Instead of directly optimizing the switching and junction times $t_{j}, j=1, \ldots, s$, one determines the arc-lengths (arc durations)

$$
\xi_{j}:=t_{j}-t_{j-1}, j=1, \ldots, s, s+1,
$$

of bang-bang and boundary arcs. Therefore, the optimization variable $z=\left(t_{1}, \ldots, t_{s}\right.$, $\left.t_{s+1}\right)^{*}$ is replaced by the optimization variable

$$
\xi:=\left(\xi_{1}, \ldots, \xi_{s}, \xi_{s+1}\right)^{*} \in \mathbb{R}^{s+1}, \xi_{j}:=t_{j}-t_{j-1} .
$$

The variables $z$ and $\xi$ are related by a linear transformation involving the regular $(s+$ 1) $\times(s+1)$-matrix $R$,

$$
\xi=R z, \quad z=R^{-1} \xi, R=\left(\begin{array}{cccc}
1 & 0 & \ldots & 0 \\
-1 & 1 & \ddots & \vdots \\
& \ddots & \ddots & 0 \\
0 & & -1 & 1
\end{array}\right) .
$$


In the arc-parameterization method, the time interval $\left[t_{j-1}, t_{j}\right]$ is mapped to the fixed interval $I_{j}:=\left[\frac{j-1}{s+1}, \frac{j}{s+1}\right]$ by the linear transformation

$$
t=a_{j}+b_{j} \tau, \tau \in I_{j}=\left[\frac{j-1}{s+1}, \frac{j}{s+1}\right],
$$

where $a_{j}=t_{j-1}-(j-1) \xi_{j}, b_{j}=(s+1) \xi_{j}$. Identifying $x(\tau) \cong x\left(a_{j}+b_{j} \tau\right)=x(t)$ in the relevant intervals, we obtain the ODE system

$$
\dot{x}(\tau)=(s+1) \xi_{j} f\left(x(\tau), u^{j}(x(\tau))\right) \text { for } \tau \in I_{j} .
$$

By concatenating the solutions in the intervals $I_{j}$ we get the continuous solution $x(t)=$ $x(t ; \xi)$ in the normalized interval $[0,1]$. When expressed via the new optimization variable $\xi$, the optimization problem $(O P)$ in 23 is equivalent to the following optimization problem $(\widetilde{O P})$ with $t_{f}=\sum_{j=1}^{s+1} \xi_{j}$ :

$$
\begin{aligned}
\operatorname{Min} \tilde{G}(\xi) & :=g\left(x(1 ; \xi), t_{f}\right), \\
\text { s.t. } \quad \widetilde{\Phi}(\xi) & :=\varphi\left(x(1 ; \xi), t_{f}\right)=0, \\
\widetilde{\mathscr{S}}(\xi) & :=S\left(x\left(\frac{k}{s+1} ; \xi\right)\right)=0 .
\end{aligned}
$$

The Lagrangian function is given by

$$
\tilde{\mathscr{L}}(\xi, \rho, \beta)=\widetilde{G}(\xi)+\rho \widetilde{\Phi}(\xi)+\beta \widetilde{\mathscr{S}}(\xi) .
$$

Using the linear transformation (27), it can easily be seen that the SSC for the optimization problems $(O P)$ and $(\widetilde{O P})$ are equivalent; cf. similar arguments in [18]. To solve this optimization problem, we use a suitable adaptation of the control package NUDOCCCS in Büskens [4[6]. Then we can take advantage of the fact that this routine also provides the Jacobian of the equality constraints and the Hessian of the Lagrangian which are needed to check the second order condition in Theorem 1

\section{Sensitivity Analysis for Bang-Bang Control Problems with a State Constraint}

The SSC given in Theorem 2 pave the way to stability and sensitivity analysis for parametric bang-bang control problems with a state constraint. Suppose that the control problem $(O C)$ in (1)-(5) depends as well on a parameter $p \in P \subset \mathbb{R}^{q}$ in the following way: Minimize

$$
J\left(x, u, t_{f}, p\right):=g\left(x\left(t_{f}\right), t_{f}, p\right)
$$

subject to the constraints on the interval $\left[0, t_{f}\right]$,

$$
\begin{aligned}
& \dot{x}(t)=f(x(t), u(t), p)=f_{0}(x(t), p)+f_{1}(x(t), p) u(t), \\
& x(0)=x_{0}, \quad \varphi\left(x\left(t_{f}\right), t_{f}, p\right)=0, \\
& u_{\min } \leq u(t) \leq u_{\max }, \\
& S(x(t), p) \leq 0 .
\end{aligned}
$$


All functions are supposed to be sufficiently smooth. This parametric control problem will be denoted by $(O C(p))$. For a fixed parameter $p_{0} \in P$, the problem $\left(O C\left(p_{0}\right)\right)$ is considered as the nominal control problem.

We shall assume that the state constraint is of order one uniformly in the parameter $p$, i.e., for

$$
S^{1}(x, u, p)=S_{x}(x, p)\left(f_{0}(x, p)+f_{1}(x, p) u\right)=a(x, p)+b(x, p) u
$$

we have $b(x, p) \not \equiv 0$ for all $p \in P$. Then the boundary control is given by

$$
u_{b}(x, p)=-a(x, p) / b(x, p) .
$$

Assuming (A1)-(A5) for the nominal control problem $\left(O C\left(p_{0}\right)\right)$, we arrive at an induced optimization problem (23) in parametric form upon inserting the parametric boundary control (38). The Lagrangian function (24) becomes

$$
\mathscr{L}(z, \rho, \beta, p)=G(z, p)+\rho \Phi(z, p)+\beta \mathscr{S}(z, p) .
$$

Using a well-known sensitivity result for finite-dimensional parametric optimization problems (cf. [7/5[6]) we arrive at the following sensitivity result for the parametric control problem.

Theorem 3. SEnSitivity ANALYSis OF THE CONTROL PROBlem $(O C(p))$.

Suppose that $\bar{u}(t)=: u\left(t, p_{0}\right)$ is a feasible control for the nominal control problem $\left(O C\left(p_{0}\right)\right)$. Assume that $u\left(t, p_{0}\right)$ has switching and junction times $\bar{t}_{j}, j=1, \ldots, s$, and a final time $\bar{t}_{f}=\bar{t}_{S+1}$ such that the SSC in Theorem 2 are satisfied. Then there exists a neighborhood $P_{0} \subset P$ of the nominal parameter $p_{0}$ and functions $t_{j}: P_{0} \rightarrow \mathbb{R}(j=$ $1, \ldots, s, s+1), \rho: P_{0} \rightarrow \mathbb{R}^{r}$ and $\beta: P_{0} \rightarrow \mathbb{R}$ with the following properties:

(1) $t_{j}\left(p_{0}\right)=\bar{t}_{j}, j=1, \ldots, s, s+1$,

(2) for every $p \in P_{0}$, the control $u(t, p)$ with switching and junction times $t_{j}(p), j=$ $1, \ldots, s$, and final time $t_{f}(p)=t_{s+1}(p)$ is a strict strong local minimum for $(O C(p))$. The values of $u(t, p)$ agree with those of the nominal control $\vec{u}(t)$ on every interior bang-bang interval and are determined on boundary arcs by $u(t, p)=u_{b}(x(t), p)$ in view of (38).

The parametric sensitivity derivatives of $z(p):=\left(t_{1}(p), \ldots, t_{s}(p), t_{s+1}(p)\right)^{*} \in \mathbb{R}^{s+1}$ and of $\rho(p), \beta(p)$ are given by the formula

$$
\left(\begin{array}{l}
d z / d p \\
d \rho^{*} / d p \\
d \beta / d p
\end{array}\right)=-\left(\begin{array}{cc}
\mathscr{L}_{z z} & \left(\Psi_{z}\right)^{*} \\
\Psi_{z} & \mathbf{0}
\end{array}\right)^{-1}\left(\begin{array}{c}
\mathscr{L}_{z p} \\
\Psi_{z p}
\end{array}\right),
$$

where we have put

$$
\Psi(z, p)=\left(\begin{array}{c}
\Phi(z, p) \\
\mathscr{S}(z, p)
\end{array}\right) .
$$

The right hand side in (40) is evaluated for the argument $(z(p), \rho(p), \beta(p))$. 
Proof. We sketch the main ideas of the proof. Since SSC hold for the nominal induced optimization problem $\left(O C\left(p_{0}\right)\right)$, the sensitivity theorem in [7|5|6] tells us that there exist a neighborhood $P_{0} \subset P$ of $p_{0}$ and, for every $p \in P_{0}$, an optimal solution and multipliers

$$
z(p)=\left(t_{1}(p), \ldots, t_{s}(p), t_{f}(p)\right), \rho(p), \beta(p),
$$

for the parametric problem $(O C(p))$. The triple $(z(p), \rho(p), \beta(p))$ satisfies the assumptions of Theorem 1 and is a $C^{1}$-function with respect to $p \in P_{0}$. We define the parametric control $u(t, p)$ in the following way. On bang-bang arcs $\left[t_{j}(p), t_{j+1}(p)\right], j \neq k$, the control $u(t, p)$ takes the values of the nominal control on the corresponding nominal $\operatorname{arcs}\left[\bar{t}_{j}, \bar{t}_{j+1}\right]$. On the boundary arc $\left[t_{k}(p), t_{k+1}(p)\right]$, the parametric control is defined by $u(t, p)=u_{b}(x(t), p)$ using the boundary control (38). Then corresponding trajectory $x(t, p)$ is determined by

$$
\dot{x}=f(x, u(t, p)), x(0, p)=x_{0} .
$$

Then the adjoint function $\lambda(t, p)$ is defined by the transversality condition

$$
\lambda\left(t_{f}(p), p\right):=(g+\rho(p) \varphi)_{x}\left(x\left(t_{f}(p), p\right), t_{f}(p)\right)
$$

and the solution to the adjoint equation

$$
\dot{\lambda}(t, p)=-H_{x}(x(t, p), \lambda(t, p), u(t, p), \eta(t, p)),
$$

where the multiplier $\eta(t, p)$ is given by the Lie-bracket

$$
\eta(t, p)=\lambda(t, p)\left[f_{1}, f\right](x(t, p), u(t, p), p) .
$$

By shrinking the set $P_{0}$ if necessary, it is easy to verify that assumptions (A1)-(A5) hold for all $p \in P_{0}$. This proves the optimality of the triple $(x(t, p), u(t, p), \lambda(t, p))$. The sensitivity formula (40) follows from standard results in [7 5[6]. Note that Theorem 3 represents an extension of a sensitivity result for bang-bang control problems without state constraints; cf. Kim, Maurer [11].

We point out that the code NUDOCCCS [4] provides also the numerical evaluation of sensitivity formula 40.

\section{Numerical Example: Time-Optimal Drug Displacement Problem}

We consider the time-optimal control problem discussed in Bell, Katusiime [3] and Maurer, Wiegand [22]. The model simulates the interaction of the two drugs, warfarin and phenylbutazone in the human blood stream. The state variables $x_{1}$ and $x_{2}$ represent the concentration of warfarin and phenylbutazone, respectively. The problem is to control the rate of infusion (control $u$ ) of the pain-killing drug phenylbutazone such 
that both drugs reach a stable steady-state in minimal time while the concentration of warfarin is bounded by a given toxic level:

$$
\begin{array}{ll}
\text { Minimize } & t_{f} \\
\text { subject to } & \dot{x}_{1}=D^{2}\left(C_{2}\left(0.02-x_{1}\right)+46.4 x_{1}\left(u-2 x_{2}\right)\right) / C_{3}, \\
& \dot{x}_{2}=D^{2}\left(C_{1}\left(u-2 x_{2}\right)+46.4 x_{2}\left(0.02-x_{1}\right)\right) / C_{3}, \\
& x_{1}(0)=0.02, \quad x_{2}(0)=0, \\
& x_{1}\left(t_{f}\right)=0.02, \quad x_{2}\left(t_{f}\right)=2, \\
& 0=u_{\min } \leq u(t) \leq u_{\max }=8 \quad \forall t \in\left[0, t_{f}\right],
\end{array}
$$

and the state constraint

$$
S(x(t))=x_{1}(t)-\alpha \leq 0 \quad \forall t \in\left[0, t_{f}\right],
$$

where

$$
\begin{aligned}
& D=D(x)=1+0.2 x_{1}+0.2 x_{2}, \\
& C_{1}=C_{1}(x)=D^{2}+232+46.4 x_{2}, \\
& C_{2}=C_{2}(x)=D^{2}+232+46.4 x_{1}, \\
& C_{3}=C_{3}(x)=C_{1} C_{2}-(46.4)^{2} x_{1} x_{2} .
\end{aligned}
$$

The augmented Hamiltonian associated with the state constraint (53) is given by

$$
H(x, u, \lambda, \eta)=\lambda f(x, u)+\eta S(x)=\frac{D^{2}}{C_{3}} \cdot K+\eta\left(x_{1}-\alpha\right),
$$

where

$$
\begin{aligned}
K:= & \lambda_{1}\left(C_{2}\left(0.02-x_{1}\right)+46.4 x_{1}\left(u-2 x_{2}\right)\right) \\
& +\lambda_{2}\left(C_{1}\left(u-2 x_{2}\right)+46.4 x_{2}\left(0.02-x_{1}\right)\right) .
\end{aligned}
$$

Then the adjoint equations 111 are

$$
\begin{aligned}
& \dot{\lambda}_{1}=-H_{x_{1}}=-\frac{\left(D^{2}\right)_{x_{1}} C_{3}-\left(C_{3}\right)_{x_{1}} D^{2}}{C_{3}^{2}} \cdot K-\frac{D^{2}}{C_{3}} \cdot K_{x_{1}}-\eta, \\
& \dot{\lambda}_{2}=-H_{x_{2}}=-\frac{\left(D^{2}\right)_{x_{2}} C_{3}-\left(C_{3}\right)_{x_{2}} D^{2}}{C_{3}^{2}} \cdot K-\frac{D^{2}}{C_{3}} \cdot K_{x_{2}},
\end{aligned}
$$

with the following partial derivatives of $K$ :

$$
\begin{aligned}
K_{x_{1}}= & 0.02 \lambda_{1}\left(C_{2}\right)_{x_{1}}-\lambda_{1}\left(C_{2}+\left(C_{2}\right)_{x_{1}} x_{1}\right)+46.4 \lambda_{1} u-92.8 x_{2} \lambda_{1} \\
& +\lambda_{2} u\left(C_{1}\right)_{x_{1}}-2 x_{2} \lambda_{2}\left(C_{1}\right)_{x_{1}}-46.4 x_{2} \lambda_{2}, \\
K_{x_{2}}= & 0.02 \lambda_{1}\left(C_{2}\right)_{x_{2}}-x_{1} \lambda_{1}\left(C_{2}\right)_{x_{2}}-92.8 x_{1} \lambda_{1}+\lambda_{2} u\left(C_{1}\right)_{x_{2}} \\
& -2 \lambda_{2}\left(C_{1}+\left(C_{1}\right)_{x_{2}} x_{2}\right)+0.928 \lambda_{2}-46.4 x_{1} \lambda_{2} .
\end{aligned}
$$


The switching function (16) is given by

$$
\sigma(x, \lambda)=H_{u}=\frac{D^{2}}{C_{3}}\left(46.4 x_{1} \lambda_{1}+C_{1} \lambda_{2}\right) .
$$

The state constraint (53) is of order one, as the function $S^{1}(x, u)$ in (6) contains the control explicitly:

$$
S^{1}(x, u)=S_{x}(x) f(x, u)=\frac{D^{2}}{C_{3}}\left(C_{2}\left(0.02-x_{1}\right)+46.4 x_{1}\left(u-2 x_{2}\right)\right) .
$$

The boundary control $u_{b}(x)$ in (7) is determined by the feedback expression

$$
u_{b}(x)=\frac{\alpha-0.02}{46.4 \alpha} C_{2}(x)+2 x_{2}
$$

Using boundary value methods it was shown in [22] that for toxic levels $0<\alpha \leq 0.0246$ the control has the following structure with two bang-bang arcs encompassing a boundary arc:

$$
u(t)=\left\{\begin{array}{lll}
8, & \text { for } & t \in\left[0, t_{1}\right), \\
u_{b}(x(t))=\frac{\alpha-0.02}{46.4 \alpha} C_{2}(x(t))+2 x_{2}(t), & \text { for } & t \in\left[t_{1}, t_{2}\right], \\
0, & \text { for } & t \in\left(t_{2}, t_{f}\right] .
\end{array}\right.
$$

We choose the toxic level $\alpha=0.024$ and compute the numerical solution using the routine IPOPT by Wächter and Biegler [27]. Using 1000 discretization points and the method of Heun for the approximation of the differential equations, IPOPT provides the solution depicted in Figs. 1, 3 The final time is computed as $t_{f}=358.731$ and the entry- and exit-time are $t_{1}=29.7747$ and $t_{2}=333.261$.

Now we solve the induced optimization problem (23) with optimization variables $z=$ $\left(t_{1}, t_{2}, t_{3}\right)$, resp., problem (30) with variables $\xi=\left(\xi_{1}, \xi_{2}, \xi_{3}\right)$, where $\xi_{1}=t_{1}, \xi_{2}=t_{2}-$ $t_{1}, \xi_{3}=t_{f}-t_{2}$. The routine NUDOCCCS [4] yields the entry-time $t_{1}=29.90806$, the exit-time $t_{2}=333.1561$ and the final time $t_{f}=358.7085$. NUDOCCCS also provides the Hessian $\tilde{\mathscr{L}}_{\xi \xi}$ and Jacobian $\tilde{\Phi}_{\xi}$,
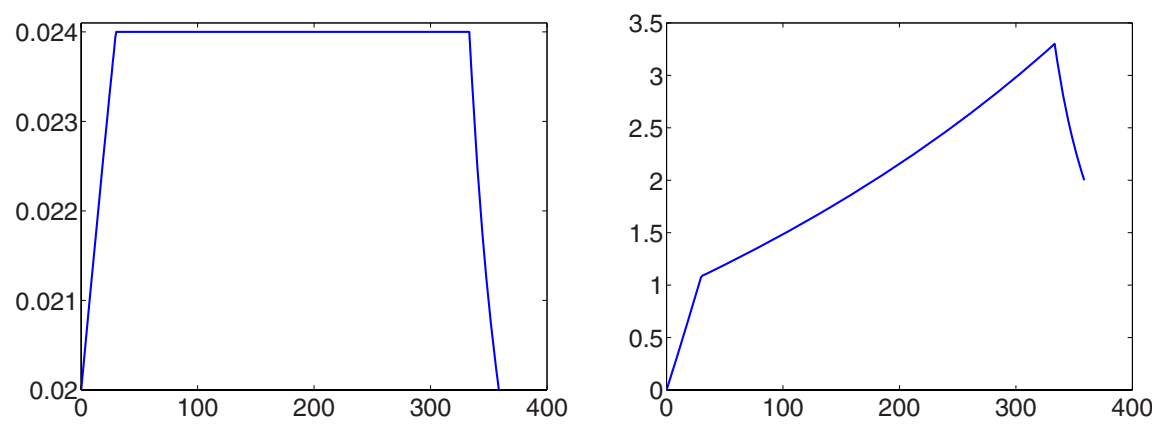

Fig. 1. Concentration of warfarin (left) and phenylbutazone (right) 

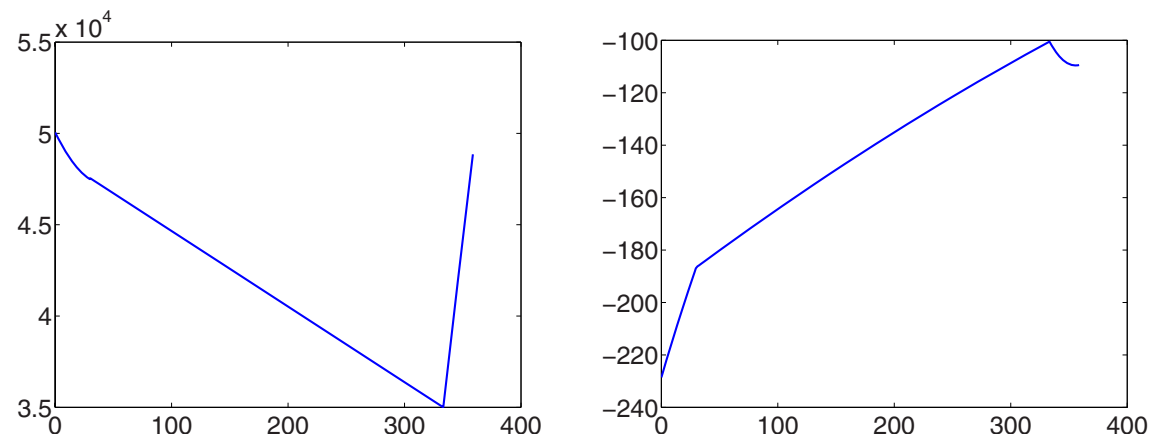

Fig. 2. Adjoint variables $\lambda_{1}(t)$ and $\lambda_{2}(t)$
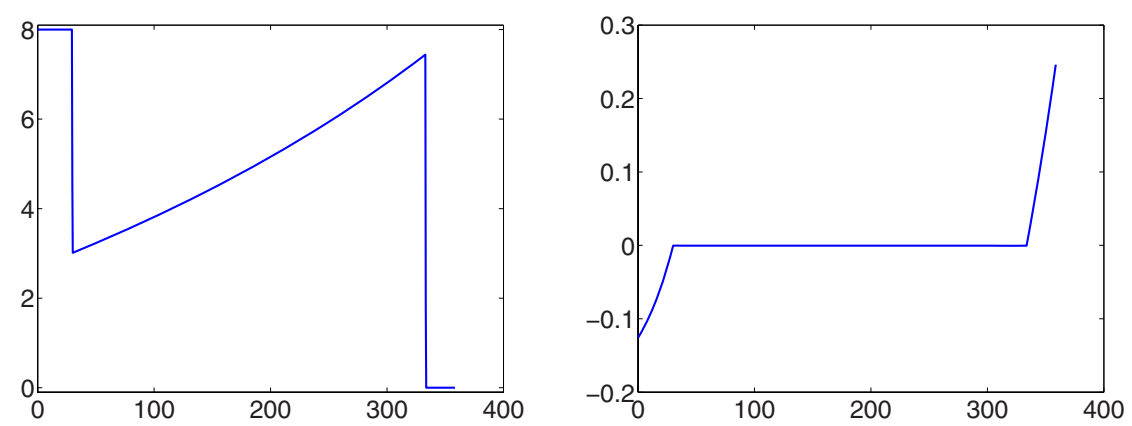

Fig. 3. Optimal control and switching function

$$
\begin{gathered}
\tilde{\mathscr{L}}_{\xi \xi}=\left(\begin{array}{rrr}
-0.046251 & -0.014521 & 0.026232 \\
-0.014521 & -0.0011993 & 0.0052637 \\
0.026232 & 0.0052637 & 0.054240
\end{array}\right), \\
\tilde{\Phi}_{\xi}=\left(\begin{array}{llr}
0.0000000 & 0.0000000 & -0.0000954 \\
0.0298122 & 0.0043075 & -0.0336047 \\
0.0001245 & 0.0000000 & 0.0000000
\end{array}\right) .
\end{gathered}
$$

Since $\operatorname{rank}\left(\tilde{\Phi}_{\xi}\right)=3$ holds, condition $(a)$ of Theorem 1 is fulfilled with $s=r=2$. Furthermore, it is clear that the first order necessary conditions hold in the induced problem, i.e., condition (b) in Theorem 1 is fulfilled. Finally, the second order condition (c) is trivially satisfied, since the matrix $\tilde{\Phi}_{\xi}$ is regular. Next, we check the regularity assumption (A1). The data provided by IPOPT give the following estimate:

$$
b(x(t))=46.4 \frac{D(x(t))^{2}}{C_{3}(x(t))} x_{1}(t) \geq 0.23 \cdot 10^{-3} .
$$

Hence, (A1) is satisfied. It can be seen in Fig. 3 that also the assumptions (A2) and (A4) hold. 


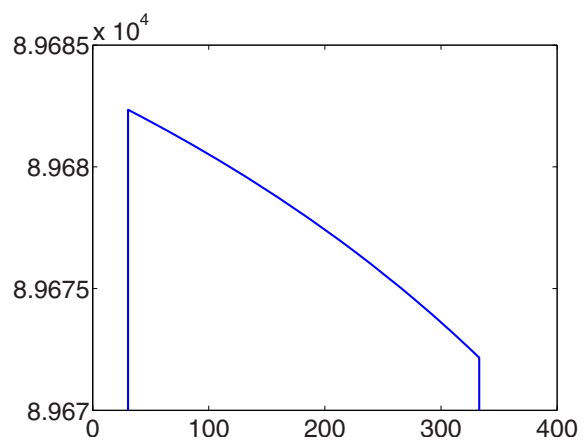

Fig. 4. Multiplier $\eta(t)$ from 73

For the verification of assumption (A5), we compute the multiplier $\eta(t)=\eta(x(t)$, $\lambda(t))$ via formula 19 ,

$$
\begin{aligned}
\eta(x, \lambda)= & \frac{2 \dot{D} C_{3}-D \dot{C}_{3}}{46.4 D C_{3} x_{1}}\left(46.4 x_{1} \lambda_{1}+C_{1} \lambda_{2}\right)+\frac{1}{46.4 x_{1}}\left(\dot{C}_{1} \lambda_{2}+C_{1} \dot{\lambda}_{2}\right)+\frac{1}{x_{1}} \dot{x}_{1} \lambda_{1} \\
& -x_{3} \cdot\left(\frac{\left(D^{2}\right)_{x_{1}} C_{3}-\left(C_{3}\right)_{x_{1}} D^{2}}{C_{3}^{2}} \cdot K+\frac{D^{2}}{C_{3}} \cdot K_{x_{1}}\right)
\end{aligned}
$$

and insert the values of $x(t), \lambda(t)$ provided by IPOPT. Note that $\eta(t) \equiv 0$ for $t \notin\left[t_{1}, t_{2}\right]$. Then Fig. 4 shows that the strict complementarity condition (A5) holds. It is noteworthy that the Lagrange multiplier for the discretized state constraint, which is provided directly by IPOPT, has the same values as the multiplier $\eta(t)$. Finally, we may conclude from Theorem 2 that the control (69) provides a strict strong minimum for the problem (47)-(53).

\section{Optimal Control for a Two-Compartment Model in Cancer Chemotherapy}

Ledzewicz, Schättler [12] considered a two-compartment model in cancer chemotherapy and established optimality using extremal field theory [13]. The state constraint (77) below has been studied in de Pinho, Ferreira, Ledzewicz, Schättler [24] using the methods developed in [13 14]. Here, we prove optimality on the basis of the SSC in Theorem 2 which allows us to apply the sensitivity result in Theorem 3 . The description of the control model is taken from [12]: "The cell cycle is broken into two compartments of which the first combines the first growth phase $G_{1}$ and the synthesis phase $S$ while the second contains the second growth phase $G_{2}$ and mitosis $M$. Let $x_{i}(t), i=1,2$, denote the number of cancer cells in the $i$-th compartment at time $t$." The control $u$ is the drug treatment which is measured by its cell-killing effect. The control problem is to minimize the cost functional with fixed final time $t_{f}$,

$$
J(x, u)=r_{1} x_{1}\left(t_{f}\right)+r_{2} x_{2}\left(t_{f}\right)+\int_{0}^{t_{f}} u(t) d t,
$$


subject to

$$
\begin{array}{ll}
\dot{x}_{1}=-a_{1} x_{1}+2(1-u) a_{2} x_{2}, & x_{1}(0)=x_{10}, \\
\dot{x}_{2}=a_{1} x_{1}-a_{2} x_{2}, & x_{2}(0)=x_{20}, \\
0 \leq u(t) \leq 1 \quad \forall t \in\left[0, t_{f}\right] . &
\end{array}
$$

The cost functional (74) can be transformed to a functional (1) of Mayer type by introducing the equation $\dot{x}_{3}=u, x_{3}(0)=0$, which yields

$$
J(x, u)=g\left(x\left(t_{f}\right)\right)=r_{1} x_{1}\left(t_{f}\right)+r_{2} x_{2}\left(t_{f}\right)+x_{3}\left(t_{f}\right) .
$$

In addition, we consider the state constraint of order one:

$$
S(x(t)):=x_{1}(t)+x_{2}(t)-\alpha \leq 0 \quad \forall t \in\left[0, t_{f}\right],
$$

which imposes an upper bound on the total number of tumor cells in both compartments. The first total time derivative (6) of $S(x)$ is given by

$$
S^{1}(x, u)=a_{2} x_{2}-2 a_{2} x_{2} u .
$$

Obviously, assumption (A1) is satisfied since $b(x(t))=-2 a_{2} x_{2}(t) \neq 0$ holds on $\left[0, t_{f}\right]$. Data for (74) and (75) are taken from [12]:

$$
r_{1}=6.94, r_{2}=3.94, a_{1}=0.197, a_{2}=0.356, t_{f}=10 .
$$

We choose the initial conditions $x_{1}(0)=x_{10}=0.86, x_{2}(0)=x_{20}=0.55$, which match approximately the solution in [12], where the terminal state $x\left(t_{f}\right), i=1,2$, was fixed and the intial state was free. The parameter $\alpha$ in the state constraint 77 will be assigned the value $\alpha=1.7$ for which the state constraint becomes active. The augmented Hamiltonian (9) is given by

$$
H=\lambda_{1}\left(-a_{1} x_{1}+2 a_{2} x_{2}\right)+\lambda_{2}\left(a_{1} x_{1}-a_{2} x_{2}\right)+\sigma u+\eta\left(x_{1}+x_{2}-\alpha\right),
$$

with switching function

$$
\sigma=\sigma(x, \lambda)=1-2 a_{2} x_{2} \lambda_{1} .
$$

The adjoint equation (11) and the transversality condition (12) yield

$$
\begin{array}{ll}
\dot{\lambda}_{1}=a_{1}\left(\lambda_{1}-\lambda_{2}\right)-\eta, & \lambda_{1}\left(t_{f}\right)=r_{1}, \\
\dot{\lambda}_{2}=a_{2}\left(2(u-1) \lambda_{1}+\lambda_{2}\right)-\eta, & \lambda_{2}\left(t_{f}\right)=r_{2} .
\end{array}
$$

The boundary control $u_{b}(x)$ satisfies the equation $S^{1}\left(x, u_{b}(x)\right) \equiv 0$ which gives

$$
u_{b}(x) \equiv \frac{1}{2}
$$

Hence, the boundary control lies in the interior of the control set and satisfies assumption (A2). The multiplier $\eta$ for the state constraint (77) is determined by equation (19): 


$$
\eta(t)=a_{1} \lambda_{1}(t)\left(\frac{x_{1}(t)}{x_{2}(t)}+1\right)-a_{2} \lambda_{1}(t)-a_{1} \lambda_{2}(t) .
$$

To determine the structure of the optimal control we first discretize the control problem with 500 gridpoints and apply the program NUDOCCCS of Büskens [4]. We find that the control has two bang-bang arcs and one boundary arc:

$$
u(t)= \begin{cases}0, & t \in\left[0, t_{1}\right), \\ u_{b}(x(t))=\frac{1}{2}, & t \in\left[t_{1}, t_{2}\right], \\ 1, & t \in\left(t_{2}, t_{f}\right] .\end{cases}
$$

Fig. 5 (left) displays the optimal control and the switching function. It clearly shows that the optimal control satisfies assumptions (A3) and (A4) since, in particular, for $k=1$ in (A4) we have $\dot{\sigma}\left(t_{1}-\right)<0$ and $\dot{\sigma}\left(t_{2}+\right)<0$. Fig. 5 (right) depicts the state constrained function $x_{1}(t)+x_{2}(t)$ and the multiplier $\eta(t)$, which is seen to satisfy the strict complementarity condition (A5). State and adjoint variables are shown in Fig. 6 resp., Fig. 7
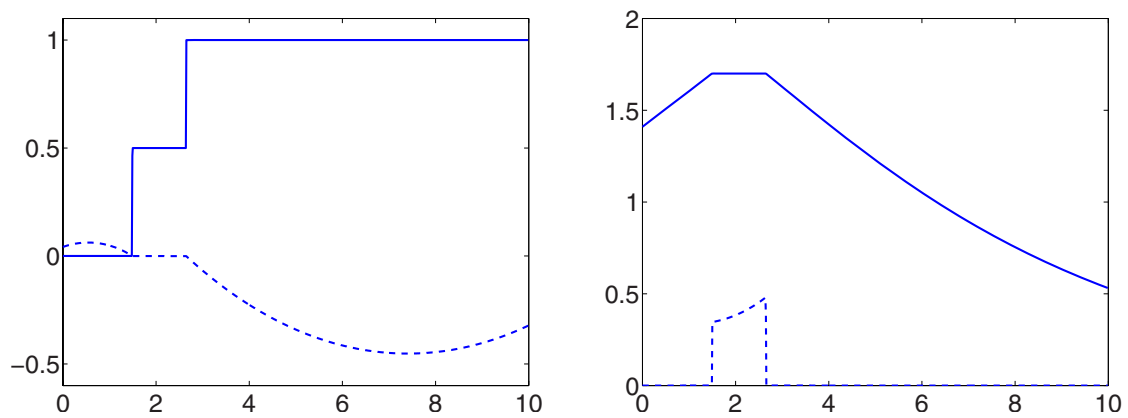

Fig. 5. Left: control with switching function (dashed); right: state constrained function $x_{1}(t)+$ $x_{2}(t)$ with multiplier $\eta(t)$ (dashed)
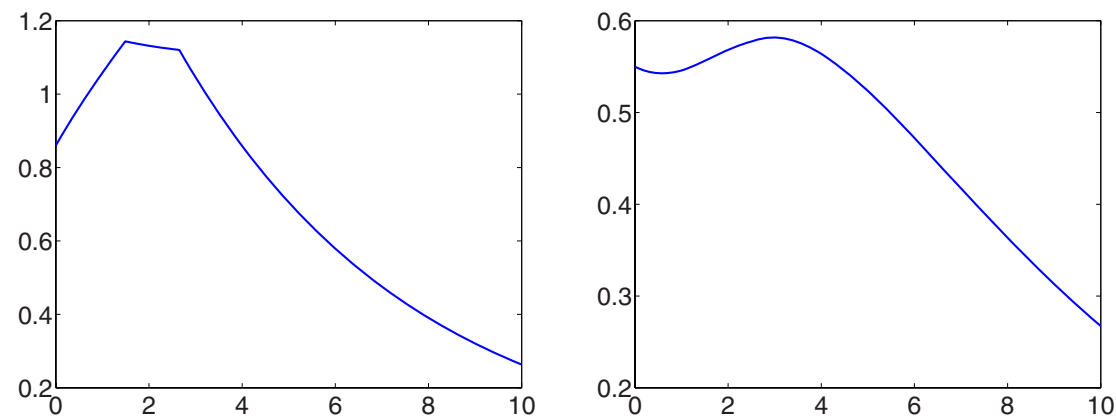

Fig. 6. State variables $x_{1}$ and $x_{2}$ 

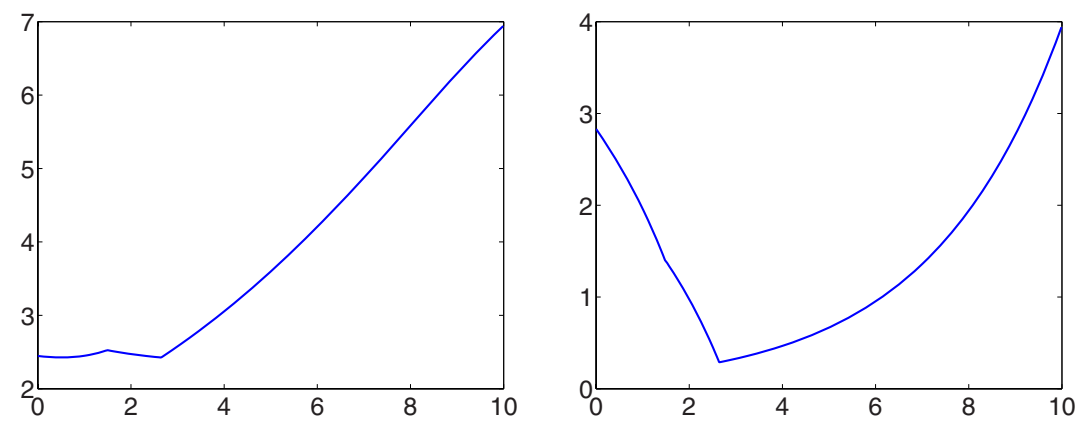

Fig. 7. Adjoint variables $\lambda_{1}$ and $\lambda_{2}$

It remains to verify the SSC in Theorem 1 for the optimization problem (30). The optimization variable is defined by (25) as

$$
\xi=\left(\xi_{1}, \xi_{2}\right)=\left(t_{1}, t_{2}-t_{1}\right) .
$$

Then the arc-length of the terminal time interval is given by $t_{f}-\xi_{1}-\xi_{2}$ with $t_{f}=10$. Since no terminal state boundary conditions are prescribed, the only equality constraint is the entry-condition of the boundary arc,

$$
x_{1}(1 / 3 ; \xi)+x_{2}(1 / 3 ; \xi)=\alpha=1.7 .
$$

The code NUDOCCCS gives the following results:

$$
\begin{aligned}
t_{1} & =1.490713, \quad t_{2}=2.653005, \\
\lambda_{1}(0) & =2.44417, \quad \lambda_{2}(0)=2.82883, \\
x_{1}\left(t_{f}\right) & =0.2635156, x_{2}\left(t_{f}\right)=0.2673589, \\
J(x, u) & =10.81033 .
\end{aligned}
$$

The Hessian of the Lagrangian for (30) is computed as

$$
\tilde{\mathscr{L}}_{\xi \xi}=\left(\begin{array}{ll}
0.225319 & 0.128060 \\
0.128060 & 0.099212
\end{array}\right)
$$

while the Jacobian of the equality constraint is given by $\tilde{\mathscr{S}}_{\xi}=(0.1979670,0)$. Obviously, the Hessian $\tilde{\mathscr{L}}_{\xi \xi}$ is positive definite and we have rank $\left(\tilde{\mathscr{S}}_{\xi}\right)=1$. Hence, we may conclude that the control (85) with data (88) satisfies the SSC in Theorem 1 and provides a strict local minimum of the optimal control problem.

Using the sensitivity result in Theorem 3 and the sensitivity formula 38, we obtain the following sensitivity derivatives for the arc-length of the first bang-bang arc and the boundary arc:

$$
\begin{aligned}
& d \xi_{1} / d a_{1}=-1.513, d \xi_{2} / d a_{1}=11.99 \\
& d \xi_{1} / d a_{2}=-3.350, d \xi_{2} / d a_{2}=0.5165 \\
& d \xi_{1} / d x_{10}=-5.359, d \xi_{1} / d x_{10}=4.421 \\
& d \xi_{1} / d x_{20}=-7.233, d \xi_{1} / d x_{20}=4.077
\end{aligned}
$$


In particular, note the high sensitivity of the boundary arc-length with respect to a variation in the parameter $a_{1}$.

Remark. Similar numerical methods can be applied to the three-compartment model for cancer chemotherapy presented in Swierniak, Ledzewicz and Schättler [25]. The model involves two control variables, a blocking agent and a recruiting agent. In this problem, it is also reasonable to consider a state constraint similar to (77). Though the analogon to Theorem 2 for vector-valued control has not been fully established in the literature, one can formulate an induced optimization problem of the type (30) and check that SSC hold; cf. Goris [8]. A paper with detailed results is in preparation.

Acknowledgements. We are indebted to Inga Altrogge [2] and Nadine Goris [8] for their numerical assistance.

\section{References}

1. Agrachev, A.A., Stefani, G., Zezza, P.L.: Strong optimality for a bang-bang trajectory. SIAM J. Control and Optimization 41, 991-1014 (2002)

2. Altrogge, I.: Hinreichende Optimalitätsbedingungen für optimale Steuerprozesse mit bangbang Steuerungen und Zustandsbeschränkungen mit verschiedenen Anwendungsbeispielen, Diploma thesis, Institut für Numerische und Angewandte Mathematik, Universität Münster (2005)

3. Bell, D.J., Katusiime, F.: A time-optimal drug displacement probem. Optimal Control Applications and Methods 1, 217-225 (1980)

4. Büskens, C.: Optimierungsmethoden und Sensitivitätsanalyse für optimale Steuerprozesse mit Steuer- und Zustands-Beschränkungen, Dissertation, Institut für Numerische Mathematik, Universität Münster (1998)

5. Büskens, C., Maurer, H.: Sensitivity analysis and real-time optimization of parametric nonlinear programming problems. In: Grötschel, M., et al. (eds.) Online Optimization of Large Scale Systems, pp. 3-16. Springer, Heidelberg (2001)

6. Büskens, C., Maurer, H.: SQP-methods for solving optimal control problems with control and state constraints: adjoint variables, sensitivity analysis and real-time control. J. of Computational and Applied Mathematics 120, 85-108 (2000)

7. Fiacco, A.V.: Introduction to sensitivity and stability analysis in nonlinear programming. In: Mathematics in Science and Engineering, vol. 165. Academic Press, New York (1983)

8. Goris, N.: Hinreichende Optimalitätsbedingungen für optimale Steuerprozesse mit Zustandsbeschränkungen und linear auftretender Steuerung: Beispiele aus der Medizin und Physik, Diploma thesis, Institut für Numerische und Angewandte Mathematik, Universität Münster (2005)

9. Hartl, R.F., Sethi, S.P., Vickson, R.G.: A survey of the maximum principles for optimal control problems with state constraints. SIAM Review 17, 181-218 (1995)

10. Kaya, C.Y., Noakes, J.L.: Computational method for time-optimal switching control. J. of Optimization Theory and Applications 117, 69-92 (2003)

11. Kim, J.-H.R., Maurer, H.: Sensitivity analysis of optimal control problems with bang-bang controls. In: Proc. of the 42nd IEEE Conf. on Decision and Control, Maui, USA, pp. 3281$3286(2003)$

12. Ledzewicz, U., Schättler, H.: Optimal bang-bang controls for a 2-compartment model in cancer chemotherapy. J. Optimization Theory and Applications 114, 609-637 (2002) 
13. Ledzewicz, U., Schättler, H.: A local field of extremals for single-input systems with state space constraints. In: Proc. of the 43nd IEEE Conf. on Decision and Control, Nassau, The Bahamas, USA, pp. 923-928 (2004)

14. Ledzewicz, U., Schättler, H.: A local field of extremals for optimal control problems with state constraints of relative degree 1. J. of Dynamical and Control Systems 12, 563-599 (2006)

15. Malanowski, K.: On normality of Lagrange multipliers for state constrained optimal control problems. Optimization 52, 75-91 (2003)

16. Maurer, H.: On optimal control problems with bounded state variables and control appearing linearly. SIAM J. Control and Optimization 15, 345-362 (1977)

17. Maurer, H.: On the minimum principle for optimal control problems with state constraints. Schriftenreihe des Rechenzentrums der Universität Münster (1977)

18. Maurer, H., Büskens, C., Kim, J.-H.R., Kaya, C.Y.: Optimization methods for the verification of second order sufficient conditions for bang-bang controls. Optimal Control Applications and Methods 26, 129-156 (2005)

19. Maurer, H., Kim, J.-H.R., Vossen, G.: On a state-constrained control problem in optimal production and maintenance. In: Deissenberg, C., Hartl, R.F. (eds.) Optimal Control and Dynamic Games, Applications in Finance, Management Science and Economics, pp. 289308. Springer, Heidelberg (2005)

20. Maurer, H., Osmolovskii, N.P.: Quadratic sufficient optimality conditions for bang-bang control problems. Control and Cybernetics 33, 555-584 (2003)

21. Maurer, H., Osmolovskii, N.P.: Second order sufficient conditions for time-optimal bangbang control problems. SIAM J. Control and Optimization 42, 2239-2263 (2004)

22. Maurer, H., Wiegand, M.: Numerical solution of a drug displacement problem with bounded state variables. Optimal Control Applications and Methods 13, 43-55 (1992)

23. Osmolovskii, N.P., Maurer, H.: Equivalence of second order optimality conditions for bangbang control problems. Part 1: Main results. Control and Cybernetics 34, 927-950 (2005);

Part 2: Proofs, variational derivatives and representations. Control and Cybernetics 36, 5-45 (2007)

24. de Pinho, M.R., Ferreira, M.M., Ledzewicz, U., Schättler, H.: A model for cancer chemotherapy with state space constraints. Nonlinear Analysis 63, 2591-2602 (2005)

25. Swierniak, A., Ledzewicz, U., Schättler, H.: Optimal control for a class of compartment models in cancer chemotherapy. Intern. J. of Applied Mathematics and Computer Science 13, 357-368 (2003)

26. Vossen, G.: Numerische Lösungsmethoden, hinreichende Optimalitätsbedingungen und Sensitivitätsanalyse für optimale bang-bang und singuläre Steuerungen, Dissertation, Institut für Numerische und Angewandte Mathematik, Universität Münster (2005)

27. Wächter, A., Biegler, L.T.: On the implementation of a primal-dual interior point filter line search algorithm for large-scale nonlinear programming. Mathematical Programming 106, 25-57 (2006) 